\title{
Upregulation of miRNA-155 expression by OxLDL in dendritic cells involves JAK1/2 kinase and transcription factors YY1 and MYB
}

\author{
HUI YAN ${ }^{*}$, SHUAI WANG ${ }^{*}$, ZHENWEI LI, WENTING ZHAO, ZHEN WANG, \\ ZEWEI SUN, YANYUN PAN and JIANHUA ZHU \\ Department of Cardiology, The First Affiliated Hospital, School of Medicine, \\ Zhejiang University, Hangzhou, Zhejiang 310003, P.R. China
}

Received July 28, 2015; Accepted March 3, 2016

DOI: $10.3892 / \mathrm{ijmm} .2016 .2526$

\begin{abstract}
Dendritic cells (DCs) have been implicated in the pathogenesis of atherosclerosis (AS). Whereas the pathogenic role of oxidized low-density lipoprotein (oxLDL) in the development and progression of AS has been recognized previously, the contribution of microRNA-155 (miR-155) to AS was previously not fully understood. It had also been noted that miR-155 levels were upregulated by oxLDL in various cell types under different (patho)physiological conditions, but its underlying mechanisms had not been examiend in detail. Thus, in the present study, we observed that oxLDL treatment increased miR-155 expression in DCs, and transfecting DCs with siRNA against scavenger receptor A (SRA) revealed that repression of SRA attenuated this upregulation. We also noted that miR-155 negatively regulated SRA expression by suppressing the JNK pathway. Furthermore, we noted that Yin Yang 1 (YY1) and V-Myb avian myeloblastosis viral oncogene homolog (MYB), which were also upregulated by oxLDL, directly bound to the cognate sequences of the promoter region of miR-155 to activate its transcription. In addition, using SP600125, a specific inhibitor for c-Jun N-terminal kinase (JNK) signaling, we demonstrated that JNK signaling was involved in the miR-155-mediated suppression of SRA expression. Thus, in the present study we uncovered the molecular mechanism through which miR-155 expression is regulated by oxLDL, and we also identified a negative feedback loop, miR-155-JNKSRA-miR-155. Our findings thus provide novel insights into the regulatory network underlying the expression and activity of miR-155 in DCs.
\end{abstract}

Correspondence to: Dr Jianhua Zhu, Department of Cardiology, The First Affiliated Hospital, School of Medicine, Zhejiang University, Hangzhou, Zhejiang 310003, P.R. China

E-mail: zjh_john@medmail.com.cn

*Contributed equally

Key words: microRNA-155, oxidized low-density lipoprotein, scavenger receptor A, Janus kinase 1/2, Yin Yang 1, MYB

\section{Introduction}

Atherosclerosis (AS) is a disease which affect the arteries and causes high mortality and morbidity in industrialized countries (1). AS was traditionally considered to be triggered by hyperlipemia, which causes multiple metabolic disturbances (2). However, other research has suggested that the initiation and development of AS involves chronic lipid-related inflammation of the vessel wall, mainly caused by activated mononuclear cells such as dendritic cells (DCs) which are present in the vascular subendothelium. The activation of DCs promoted the progression of atherosclerotic plaques by affecting cellular recruitment, interaction and secretory products (3). Thus, DCs play an important role in triggering development of AS.

MicroRNAs (miRNAs) are single-stranded RNAs and are approximately 22 nucleotides in length. In a sequence-specific manner, miRNAs induce the formation of silencing complexes that lead to translational repression and/or mRNA decay in target genes (4). miRNAs have been shown to be involved in a variety of physiological and pathological activities and the development of various conditions and diseases, including hypertrophic remodeling, heart failure and arrhythmia (5-10). Previous research has shown that a number of miRNAs are involved in mediating the functions of DCs and thus play important roles in the pathogenesis of AS (11). miR-155 is encoded within an exon of a non-coding RNA transcribed from the B-cell integration cluster (BIC), which is located on chromosome 21 (12). Previous research has indicated that miR-155 is involved in regulating various biological processes, including hematopoietic differentiation, viral infection, AS and tumorigenesis (11,13-16). Several previous studies have also demonstrated that miR-155 is involved in various immune-mediated inflammatory diseases although the exact role of miR-155 in the vasculogenesis, development and progression of AS remains a matter of debate. For instance, Nazari-Jahantigh et al (17) and other researchers have reported that miR-155 promotes the development of AS (18). On the other hand, research from our laboratory suggests that miR-155 plays a protective role in the development and progression of AS (19). In a murine model of hyperlipidemia, miR-155 was found to act as an anti-inflammatory and protective factor against AS (20).

Despite those controversial reports regarding the role of miR-155 in AS, it has been reported in a number of studies that treatment with oxidized low-density lipoprotein (oxLDL), which 
plays a well-known pathogenic role in the development and progression of AS, induces the expression of miR-155 in various cell types under different (patho)physiological conditions (21-23). However, exactly how miRNA-155 was regulated in DCs treated with oxLDL was not fully understood. The aim of the present study was thus to investigate the molecular mechanisms by which miR-155 expression is mediated by oxLDL in DCs.

\section{Materials and methods}

Cell culture. In the present study, experiments using human peripheral blood were undertaken according to the principles of the Declaration of Helsinki and were approved by the Ethics Committee of Zhejiang University (Hangzhou, China). We also obtained appropriate consent from the healthy volunteer donors. Human peripheral blood was obtained from healthy volunteer adults for subsequent isolation of monocytes using a lymphocyte separation liquid system (Sigma-Aldrich, St. Louis, MO, USA). Cells were cultured in complete medium [RPMI-1640, 10\% fetal calf serum and 1,000 U/ml granulocyte-macrophage colony-stimulating factor (GM-CSF)] and 1,000 U/ml interleukin-4 (IL-4) (all from Peprotech, Inc., Rocky Hill, NJ, USA) for 5 days. Complete medium was replaced every other day. oxLDL (40 $\mu \mathrm{g} / \mathrm{ml})$ (Yiyuan Biotechnology, Guangzhou, China) was added at day 5, and cells were harvested at day 7. All inhibitors, namely SB203580, SP600125, UO126, PDTC and AG490 (all purchased from Sigma-Aldrich), were added $30 \mathrm{~min}$ prior to oxLDL (40 $\mu \mathrm{g} /$ $\mathrm{ml}$; Yiyuan Biotechnology) treatment for $24 \mathrm{~h}$. Dimethyl sulphoxide (DMSO; Sigma-Aldrich) was used as the control of inhibitors since these chemical inhibitors were dissolved in DMSO. Finally, the cells were harvested for later experiments.

siRNA transfection. DCs were transfected with siRNA against scavenger receptor A (SRA), lectin-like oxidized low-density lipoprotein (LDL) receptor-1 (LOX-1), cluster determinant 36 (CD36), signal transducer and activator of transcription 3 (STAT3), miR-155 inhibitor, Yin Yang 1 (YY1) or V-Myb avian myeloblastosis viral oncogene homolog (MYB) $(50 \mathrm{nM})$ (Baiao Biotech, Inc., Changchun, China) using Lipofectamine RNAiMAX reagent (Life Technologies, Grand Island, NY, USA) according to the manufacturer's instructions. Control siRNA against no significant targets (Baiao Biotech, Inc.) was also used as the negative control (NC). Briefly, $2 \mu \mathrm{l}$ siRNA, at a final concentration of $50 \mathrm{nM}$, and $5 \mu \mathrm{l}$ transfection reagent were mixed and added to DCs in serum-free medium. Cells were harvested $24 \mathrm{~h}$ post-transfection.

Reverse transcription-quantitative PCR (RT-qPCR). Total RNA was isolated using an miRcute miRNA isolation kit (Tiangen Biotech Co., Ltd., Beijing, China). cDNA was generated using a PrimeScript miRNA RT reagent kit, and qPCR was carried out using SYBR-Green Premix Ex Taq (both from Takara Bio, Inc., Otsu, Japan). PCR was performed using an ABI PRISM 7500 Sequence Detection system (Applied Biosystems Life Technologies, Foster City, CA, USA). U6 was used as an endogenous control. The sequences of primers used for qPCR are: U6 forward, 5-ACTTGCTCATCAAGGTGT CAG-3' and reverse, 5-TGACCAGCGTTTGTTCAATGT-3'; miR-155 forward, 5-UUAAUGCUAAUCGUGAGAGGGGU-3' and reverse uni-miR qPCR primer, 5-TTTTTTTTTTTTTTT TTTTT-3' (Takara Bio, Inc.).

Western blot analysis. DCs were lysed and a total of $60 \mu \mathrm{g}$ protein lysates were subjected to electrophoresis on $10 \%$ polyacrylamide SDS gel,followed by transfer onto PVDF membranes (Millipore Corp., Billerica, MA, USA). The membranes were subsequently probed using primary antibodies: rabbit antiYY1 (1:1,000; \#2185), rabbit anti-MYB (1:1,000; \#12319) and rabbit anti-Janus kinase 1/2 (JAK1/2) (1:1,000; H-103) (all from Cell Signaling Technology, Beverly, MA, USA) or rabbit antiGAPDH (1:1,000; CW101M) (Changwei, China). An ECL kit (Pierce Biotechnology, Inc., Rockford, IL, USA) was then used to detect chemiluminescence.

Dual-luciferase reporter assay. Genomic DNA was extracted from DCs using a Genomic DNA Extraction kit (Kangwei, Beijing, China). Promoter regions of miRNA-155, 2,000, 1,500 or $1,000 \mathrm{bp}$ upstream of the transcription initiation site were amplified using PCR. The PCR products were gel-purified and then subcloned into a pGL3 luciferase reporter vector (Promega Corp., Madison, WI, USA) at HindIII and SacI sites. The sequences of the primers used to subclone these cis-regulatory elements are shown below: 2k-promoter forward, 5'-CAAGCACTGCCGACTACAATA-3' and reverse, 5'-CCA GGCTGATTCATCCCACTG-3'; 1.5k-promoter forward, 5'-GCCGACTCAGGCACTACAATA-3' and reverse, 5'-CCA GGCTGATTCATCCCACTG-3'; and 1k-promoter forward, 5'-GACTACAATCACGAACTGCCA-3' and reverse, 5'-CCA GGCTGATTCATCCCACTG-3'. The reporter was transfected into DCs together with transcription factors (TFs) of interest and activity was determined $48 \mathrm{~h}$ post-transfection. Firefly luciferase activity was also measured using a dual-luciferase reporter assay system (Promega Corp.). Relative reporter activity was subsequently obtained by normalization to Renilla control luciferase activity.

TF filter plate assay. After treatment, DCs were harvested to extract nuclear proteins. A TF filter plate assay (Signosis Inc., Sunnyvale, CA, USA) was performed according to the manufacturer's instructions. In brief, a TF DNA complex was mixed and subsequently separated from free probes, prior to elution of bound probes. Hybridization of the eluted probe with a hybridization plate was then performed and finally detected using a luminometer (BioTek, Winooski, VT, USA).

Co-immunoprecipitation (Co-IP) assay. Protein lysates were purified from DCs, as mentioned above. One milligram of protein lysate was incubated with $5 \mu \mathrm{g}$ of the appropriate antibody [anti-MYB (\#12319) or rabbit IgG (\#14708); Cell Signaling Technology] on a rotator (JinDun, Ningbo, China), at $4^{\circ} \mathrm{C}$, overnight, followed by further incubation for a 2-4-h period with $10 \mu \mathrm{l}$ protein A-agarose beads (Cell Signaling Technology). After being washed three times, the immunocomplexes were precipitated by centrifugation at 3,000 rpm for $3 \mathrm{~min}$ at $4^{\circ} \mathrm{C}$. The bound proteins were released with $2 \mathrm{X}$ SDS buffer, boiled for $5 \mathrm{~min}$, and subjected to western blot analysis.

Bioinformatics analysis. For the purposes of the present study, the promoter sequence of miR-155 was obtained from the 

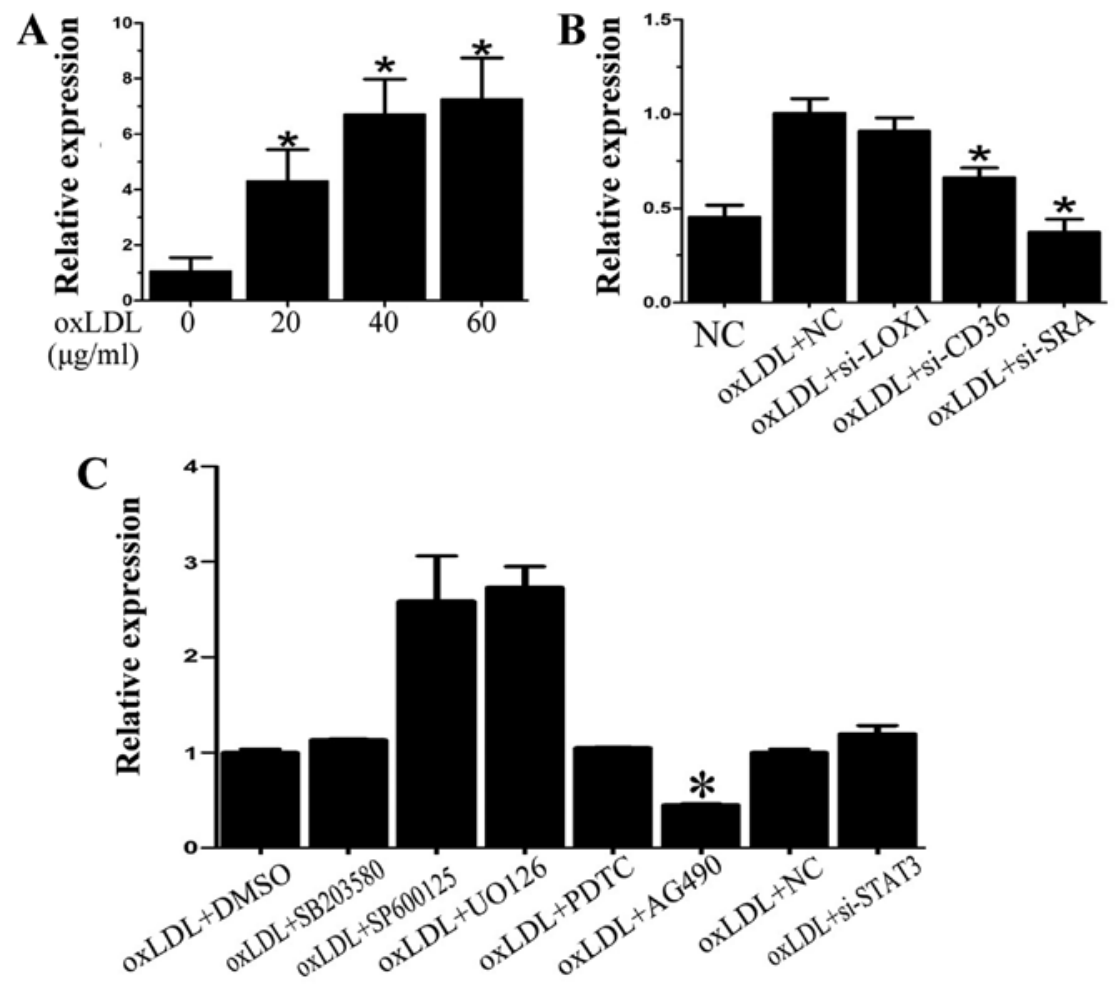

Figure 1. miR-155 expression is upregulated by oxidized low-density lipoprotein (oxLDL) through scavenger receptor A (SRA) and Janus kinase 1/2 (JAK1/2) signaling. (A) miR-155 was increased in dendritic cells (DCs) following oxLDL treatment. (B) Knockdown of cluster determinant 36 (CD36) or SRA both decreased miR-155 expression induced by oxLDL. (C) p38, c-Jun N-terminal kinase (JNK), extracellular signal-regulated kinase (ERK)1/2 and nuclear

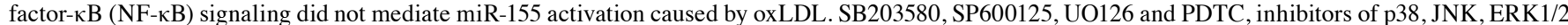
and NF- $\mathrm{kB}$, respectively, were applied before oxLDL treatment. Signal transducer and activator of transcription 3 (STAT3) siRNA also exerted almost no effect on miR-155 expression. AG490, the inhibitor of JAK2, significantly decreased oxLDL-induced miR-155 expression. Data are presented as the means \pm SD. $n=5$ and "p<0.05, compared with control group [negative control (NC) or oxLDL + DMSO].

website http:/genome.ucsc.edu/cgi-bin/hgNear. Transcription factor binding sites were predicted using the website http://www. cbrc.jp/research/db/TFSEARCH.html by typing the promoter sequence of miR-155.

Chromatin immunoprecipitation (ChIP) assay. DCs were cross-linked with $1 \%$ formaldehyde in medium for $10 \mathrm{~min}$ at $25^{\circ} \mathrm{C}$ and washed with ice-cold PBS before resuspension in $1 \mathrm{ml} \mathrm{SDS} \mathrm{lysis} \mathrm{buffer.} \mathrm{The} \mathrm{cell} \mathrm{suspension} \mathrm{was} \mathrm{sonicated} \mathrm{to}$ average 300-900-nt length DNA fragments before being precleared with $20 \mu \mathrm{l}$ protein A-agarose beads for $30 \mathrm{~min}$ at $4^{\circ} \mathrm{C}$. After the beads were removed, the chromatin solution was immunoprecipitated with an antibody (anti-YY1 or anti-MYB) at $4^{\circ} \mathrm{C}$ overnight, followed by incubation with protein $\mathrm{A}$-agarose beads for an additional $1 \mathrm{~h}$ at $4^{\circ} \mathrm{C}$. The immune complex was then eluted using $150 \mu \mathrm{l}$ elution buffer (1\% SDS and $0.1 \mathrm{M}$ $\mathrm{NaHCO}_{3}$ ). Formaldehyde cross-linking was reversed by heating at $65^{\circ} \mathrm{C}$ for $4 \mathrm{~h}$ with proteinase $\mathrm{K}$ and $\mathrm{NaCl}(5 \mathrm{M})$. Genomic DNA was purified from the immunoprecipitate and analyzed by PCR using specifically designed primers to detect target regions of interest. Primers to amplify the region of YY1-a were: forward, 5'-GTTCCAACACAAACTCTT-3' and reverse, 5'-CTCTAATCAGGCAATTCG-3'; primers to amplify the region of 'YY1-b' were: forward, 5'-CTT ATTATATAAAGGCGC-3' and reverse, 5'-GGTGAC CCATCACGAAAGG-3'; primers to amplify the region of 'MYB' were: forward, 5'-GACACAGTCTTACGTTCGA-3' and reverse, 5'-CGACCTCACTGTCGTATA-3'.
Statistical analysis. Statistically significant differences between groups were calculated using unpaired t-tests. Values are expressed as the means $\pm \mathrm{SD}$, and a $\mathrm{p}$-value $<0.05$ was considered to indicate a statistically significant difference. Every experiment was performed at least 5 times $(n \geq 5)$.

\section{Results}

miR-155 is upregulated by oxLDL through modulation of SRA and CD36. miR-155 has been established as an inflammatory-related miRNA, and its role in AS has been examined; consistent with our previous research (19), we found that miR-155 was increased in DCs in response to oxLDL treatment in a dose-dependent manner (Fig. 1A). Subsequently, to explore the molecular basis underlying this upregulation, we used siRNA to knock down three scavenger receptors, SRA, CD36 and LOX-1, all of which are known to mediate endocytosis of oxLDL in macrophages (24), and then tested whether any of these scavenger receptors was involved in the upregulation of miR-155 by oxLDL in DCs. As shown in Fig. 1B, siRNA knockdown of either CD36 or SRA, but not LOX-1, significantly decreased the levels of miR-155 induced by oxLDL. Thus, we conclude that CD36 and SRA are involved in the oxLDL-mediated miR-155 upregulation in DCs.

miR-155 is upregulated by oXLDL and the JAK1/2 signaling pathway is involved. Subsequently, we sought to probe the potential pathways by which oxLDL triggers miR-155 tran- 
A

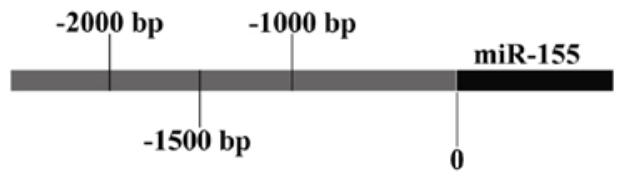

B

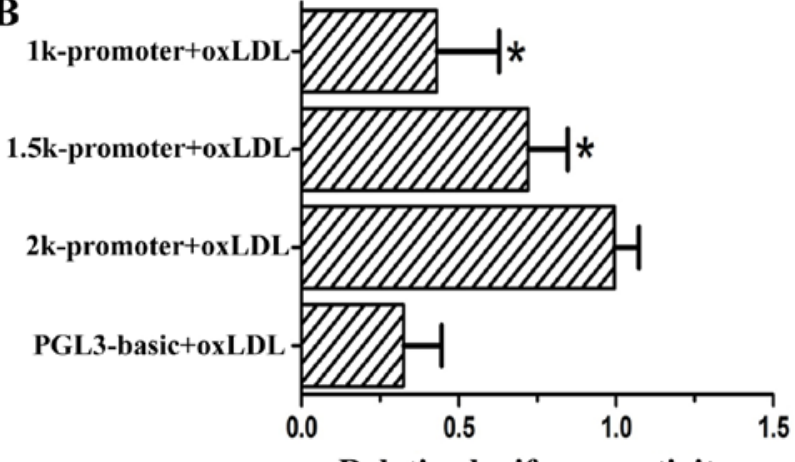

Relative luciferase activity

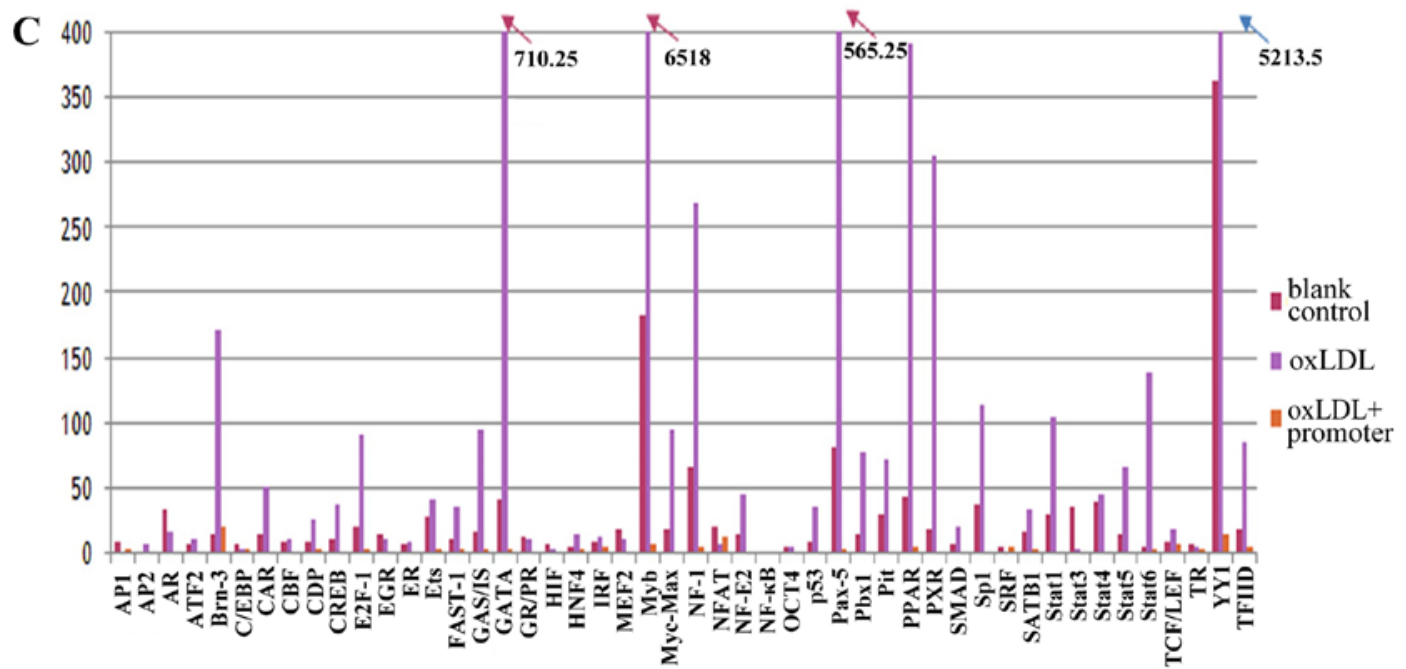

Figure 2. Transcription factors (TFs) Yin Yang 1 (YY1) and V-Myb avian myeloblastosis viral oncogene homolog (MYB) participate in miR-155 transcriptional regulation. (A) Schematic representation of the promoter regions that were subcloned into pGL3-basic luciferase reporter. (B) Activation of the promoter-luciferase reporters by oxidized low-density lipoprotein (oxLDL) in dendritic cells (DCs). (C) TF filter plate assay revealed the robust binding of YY1 and MYB on miR-155 promoter region. Data are presented as the means \pm SD. n=5, and " $\mathrm{p}<0.05$ vs. pGL3-basic + oxLDL.

scription. Since mitogen-activated protein kinase (MAPK) and nuclear factor- $\mathrm{\kappa B}(\mathrm{NF}-\mathrm{\kappa} \mathrm{B})$ pathways have been established as key signaling pathways driving inflammatory responses, we pre-treated DCs with specific inhibitors for $\mathrm{p} 38$, c-Jun N-terminal kinase (JNK), extracellular signal-regulated kinase (ERK) $1 / 2$ or NF- $\kappa B$ prior to oxLDL treatment. Neither MAPK nor NF- $\kappa B$ signaling was directly involved in mediating miR-155 activation caused by oxLDL (Fig. 1C). Since previous research has postulated a potential role of JAK and STAT3 in miR-155 transcription (25), we then examined whether they were involved in oxLDL-induced miR-155 upregulation using specific inhibitors for JAK and STAT3. The inhibition of JAK2 by AG490 significantly reduced the induction of miR-155 in response to oxLDL, but inhibition of STAT3 using STAT3 siRNA did not exert any significant effect on miR-155 expression induced by oxLDL (Fig. 1C).

TFs YYI and MYB participate in miR-155 transcriptional regulation. To study the regulatory elements in the promoter of miR-155, we constructed a series of luciferase reporter plasmids including $1,000,1,500$ and 2,000 bp upstream of the transcription initiation site of miR-155 (Fig. 2A), as previously described (26). Data from the dual luciferase reporter assays showed that the 2,000 bp region exhibited the highest activity in response to oxLDL treatment, whereas the $1,000 \mathrm{bp}$ sequence showed the lowest, but still significant, activation (Fig 2B). Subsequently, we performed TF filter plate assays using the 2,000 bp sequence of miR-155 promoter. As shown in Fig. 2C, YY1 and MYB had more significant bindings to this promoter region than other $46 \mathrm{TFs}$ examined.

YY1 and MYB promote miR-155 transcription by directly binding to the miR-155 promoter. We next attempted to elucidate whether YY1 and/or MYB was involved in oxLDL-induced miR-155 transcription in DCs. By transfecting cells with siRNA against YY1 and MYB, we demonstrated that knockdown of both YY1 and MYB reduced baseline miR-155 expression both on their own and also with oxLDL treatment (Fig. 3A and B), suggesting that YY1 and MYB regulated miR-155 expression both at basal levels and in response to oxLDL. Bioinformatics analysis revealed two potential binding sites for YY1 and one for MYB on the miR-155 promoter (Fig. 3C), and we confirmed that oxLDL treatment strengthened the binding of YY1 to the first binding site (Fig. 3D), and that oxLDL also induced binding between MYB and the miR-155 promoter (Fig. 3D). Taken together, our results suggest that both YY1 and MYB 


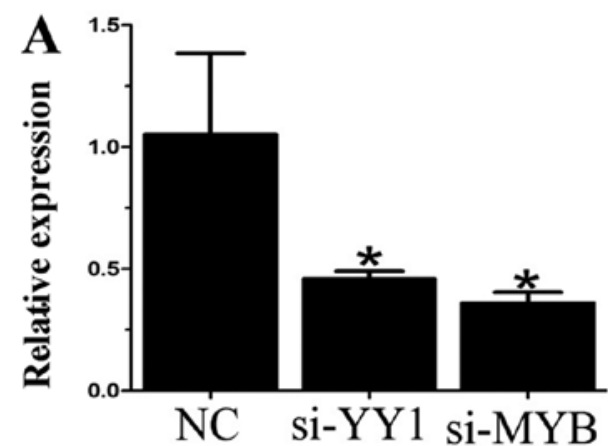

C YY1:

\section{a: TGTGTGACATGTTTTAT b: GAGACCACATACTTGA}

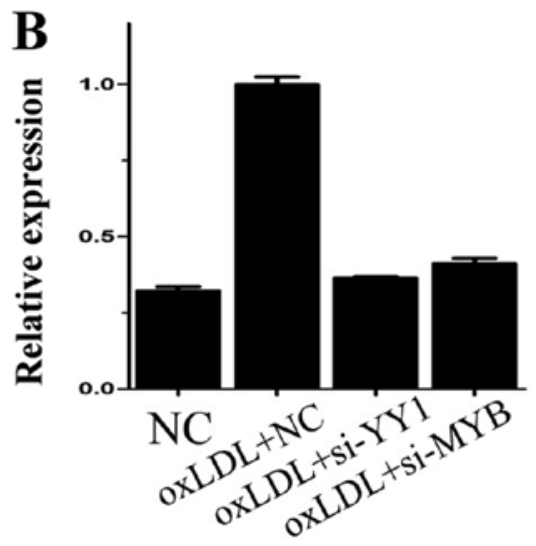

MYB:

\section{AAAGGTAACAATGTC}

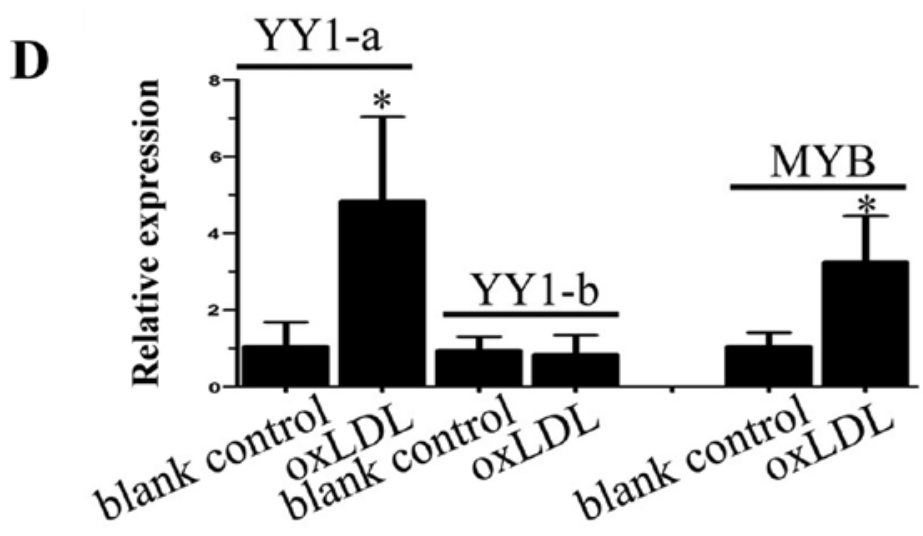

Figure 3. Yin Yang 1 (YY1) and V-Myb avian myeloblastosis viral oncogene homolog (MYB) promote miR-155 transcription by directly binding to miR-155 promoter. (A) miR-155 expression was decreased by transfection with siRNA specifically against YY1 or MYB. (B) The oxidized low-density lipoprotein (oxLDL)-induced increase in miR-155 expression was attenuated by transfection with siRNA against YY1 or MYB. (C) Schematic depiction of potential binding sites in the miR-155 promoter for YY1 and MYB. Two binding sites of YY1 and one binding site of MYB are shown (underlined). (D) OxLDL treatment strengthened the binding of YY1 to the first YY1 binding site, and the binding of MYB to its cognate site in the miR-155 promoter. Data are presented as the means \pm SD. $n=5 /$ group. " $p<0.05$ vs. control [blank control or negative control $(\mathrm{NC})$ ].

mediate miR-155 expression via directly binding to the cisregulatory sequence of miR-155.

$Y Y 1$ and MYB cooperatively induce miR-155 expression through JAK1/2 kinase activation. To further examine whether YY1 and MYB were also activated by JAK1/2 or other kinases, we firstly tested whether the expression of YY1, MYB and JAK1/2 was altered by oxLDL treatment. Western blot analysis showed that YY1, MYB and JAK1/2 were increased following treatment with oxLDL, in a dose-dependent fashion (Fig. 4A). Inhibition of JAK1/2 using AG490 abolished oxLDL-induced promotion of YY1 and MYB expression (Fig. 4B). Moreover, we observed the interaction between YY1 and MYB, which was enhanced by oxLDL treatment (Fig. 4C). Thus, we conclude that YY1 and MYB cooperatively induce miR-155 expression through JAK1/2 kinase.

Negative feedback and the induction of miR-155 by oxLDL. We observed that oxLDL promoted miR-155 expression partially through binding to SRA. Our previous research had shown that
miR-155 inhibited SRA expression and the JNK pathway (24), and JNK signaling has been demonstrated to control SRA expression in macrophages. Thus, we questioned whether miR-155 inhibited SRA expression through suppressing the JNK pathway. Western blot analysis showed that miR-155 inhibition upregulated SRA whereas SP600125, a specific JNK inhibitor, abrogated the promoting effect caused by miR-155 inhibitor on SRA expression (Fig. 4D). Thus, a negative feedback loop exists between miR-155-SRA-JNK-miR-155 in response to oxLDL, as is illustrated in Fig. 5.

\section{Discussion}

Previous research has demonstrated that miR-155 is involved in numerous biological processes, including haematopoietic lineage commitment and tumor formation $(13,27,28)$. $\mathrm{BIC} / \mathrm{miR}-155$ expression is greatly increased in activated $\mathrm{B}$ and T cells and DCs (29). Previous research has revealed that miR-155-deficient mice exhibited impaired antigenpresenting capacities (27). Furthermore, increased expression 
A

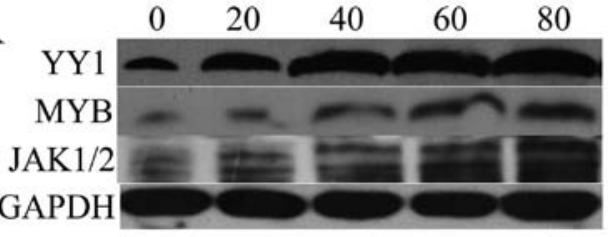
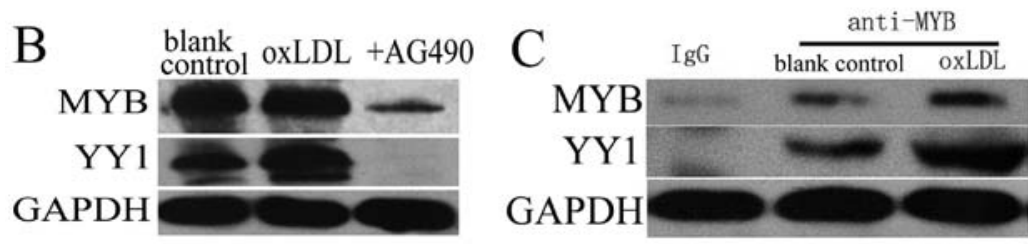

$\mathrm{D}$

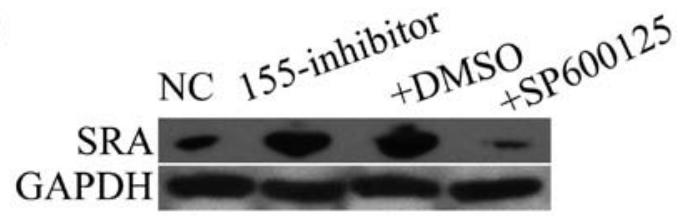

Figure 4. Janus kinase 1/2 (JAK1/2) signaling is involved in mediating miR-155 expression through Yin Yang 1 (YY1) and V-Myb avian myeloblastosis viral oncogene homolog (MYB). (A) Expression levels of YY1, MYB and JAK1/2 were increased following oxidized low-density lipoprotein (oxLDL) treatment. (B) Inhibiting JAK1/2 signaling by AG490 reduced the expression levels of MYB and YY1. (C) Physical association between MYB and YY1 was potentiated by oxLDL treatment as revealed by co-immunoprecipitation assay. (D) miR-155 inhibition upregulated scavenger receptor A (SRA) expression, while SP600125 treatment abolished the miR-155 inhibition-induced SRA expression. Data are presented as the means \pm SD. $n=5$. " $p<0.05$.

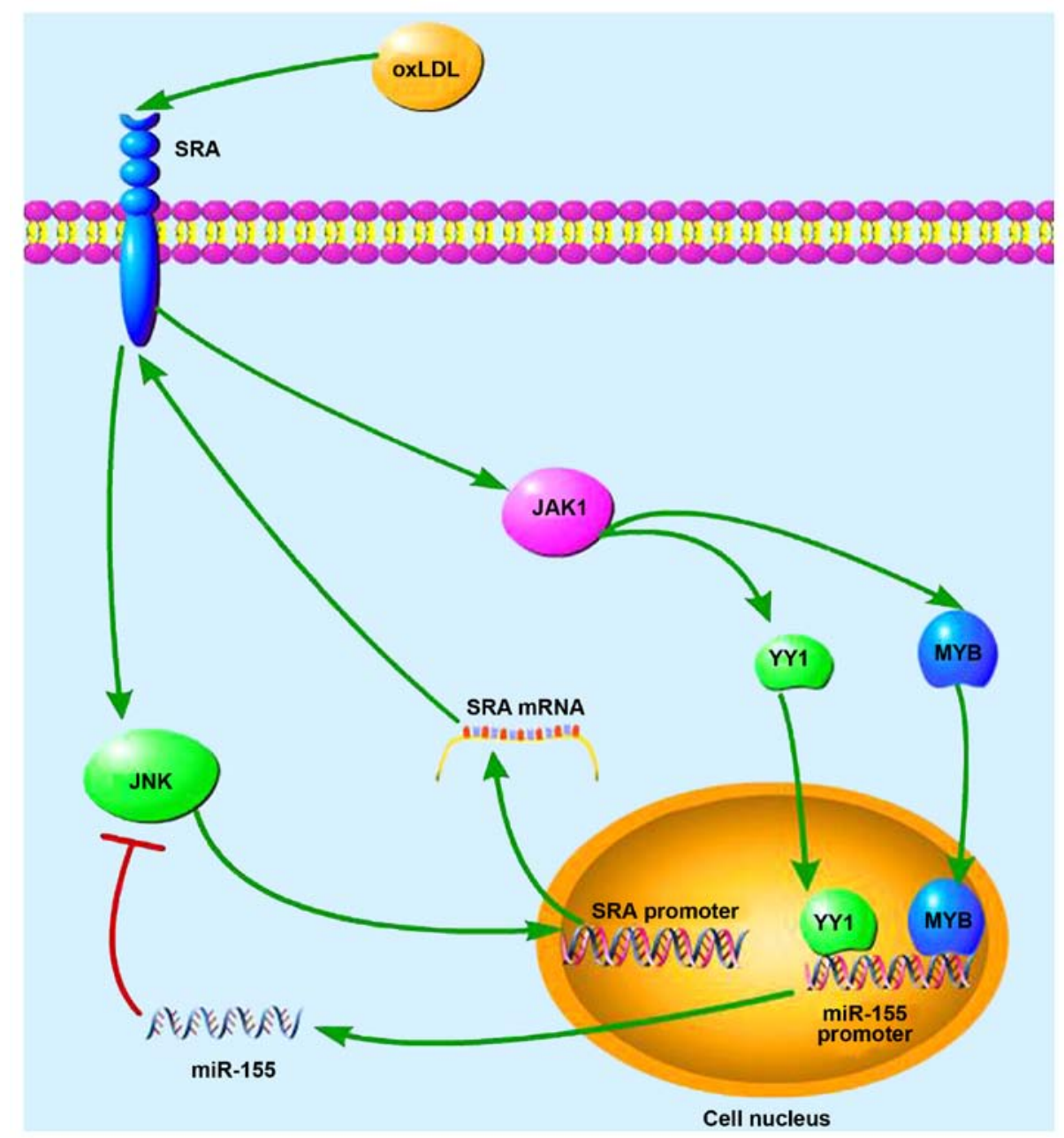

Figure 5. Schematic representation of a negative feedback loop miR-155-SRA-JNK-miR-155 involved in mediating miR-155 expression by oxidized lowdensity lipoprotein (oxLDL). YY1, Yin Yang 1; MYB, V-Myb avian myeloblastosis viral oncogene homolog; SRA, scavenger receptor A; JAK1, Janus kinase 1; JNK, -Jun N-terminal kinase. 
of BIC/miR-155 was observed in patients with Hodgkin's lymphoma (30) and miR-155 was found to be overexpressed in the bone marrow of patients with certain subtypes of acute myeloid leukemia (AML) (13), suggesting that miR-155 is linked to human diseases. Indeed, miRNA-155 has been shown to play specific roles in regulating various diseases and may thus be a good candidate for predicting clinical prognosis and generating potential therapeutic treatments for a number of diseases, including renal cell carcinoma (15), and breast (16) and colon cancer (28). Moreover, miR-155 appears to be involved in biochemical mechanisms associated with viral infections. For example, miR-155 contributes to Epstein-Barr virus (EBV) immortalization by regulating $\mathrm{NF}-\kappa \mathrm{B}$ signaling and suppressing the host innate immunity to latent viral infection (14). Therefore, miR-155 exhibits a wide spectrum of physiological and pathological function.

Interestingly, miR-155 has also been found to be involved in cardiovascular disease: overexpression of this miRNA-155 was accompanied by impaired angiotensin II type 1 receptor (AT1R) activity and low blood pressure (31). Our previous research has shown that miR-155 contributed to the prevention of AS development and progression (19). Although previous research has demonstrated the role of miR-155 in multiple diseases, relatively little is known about how its expression is mediated in a disease setting, particularly in an immuno-inflammatory context.

Several studies have shown that the increase in miR-155 expression is related to the ERK and JNK signaling pathways (32). For example, B-cell receptor activation induced BIC/miR-155 expression via a conserved AP-1 element (32). miR-155 expression was also induced in response to LPS, resulting in a decrease in Src homology domain 2 (SH2)containing inositol phosphatase (SHIP1) expression and allowing PI3K activation of $\mathrm{NF}-\kappa \mathrm{B}$ and MAPK to proceed and promote the pro-inflammatory response (33). However, in the presence of interleukin (IL)-10, miR-155 expression was inhibited through the STAT3 signaling pathway, which led to the recovery of SHIP1 expression and the conversion of phosphatidylinositol $(3,4,5)$-trisphosphate (PIP3) back to its inactive phosphatidylinositol (4,5)-bisphosphate (PIP2) state (25). As a result, pro-inflammatory responses were switched off (25). Indeed, previous data from our laboratory showed that miR-155 contributed to the prevention of inflammation and its progression by regulating the MAPK pathway via specifically targeting mitogen-activated protein kinase kinase kinase 10 (19).

In the present study, neither MAPK, NF- $\kappa \mathrm{B}$ nor STAT3 pathways were found to be involved in mediating the expression of miR-155, which was upregulated in response to oxLDL. On the contrary, we demonstrated that oxLDL promoted miR-155 expression through the SRA receptor and JAK2 signaling pathway, as the inhibition of SRA or JAK1/2 signaling significantly decreased the miR-155 levels which had risen in response to oxLDL. Moreover, miR-155 negatively regulated SRA expression by suppressing the JNK pathway. Furthermore, we identified response elements present in the miR-155 promoter region that mediated the activity of the cisregulatory sequence of miR-155. These response elements were recognized by the transcription factors YY1 and MYB, both of which exhibited physical interaction which was potentiated by oxLDL stimulation. Interestingly, YY1 and MYB participated in mediating miR-155 transcription by binding to the miR-155 promoter through JAK1/2 kinase. Taken together, the results of our present study demonstrated the molecular mechanisms by which miR-155 was regulated upon oxLDL treatment. Given that oxLDL is an important promoter of the development of AS, and DCs are also critically involved in the inflammatory reaction that occurs during AS initiation and progression, our findings provide novel insights into how miR-155 expression and activity is mediated in DCs during the course of AS, in which YY1 and MYB may also play an important regulatory role. Consistent with these findings, YY1 was previously shown to be induced by vascular cell injury (34), whereas MYB was also involved in AS (35). However, we do not rule out the possibility that other TFs also play a role in mediating miR-155 transcription. It will also be interesting to further explore more roles of miR-155 in DC functions, and clarify the exact role of miR-155 in the development of AS.

In conclusion, our study revealed that oxLDL promotes miR-155 expression partially through the SRA receptor and the JAK2 signaling pathway in human peripheral blood-derived DCs. oxLDL induces the expression of TFs MYB and YY1, which subsequently bind to the regulatory sequence of miRNA-155 to activate its transcription. Furthermore, in the present study we uncovered a negative feedback loop miR-155-SRA-JNK-miR-155 involved in the activation of miR-155 by oxLDL. Therefore, our findings provide novel insights into the mechanisms underlying the increased expression of miR-155 by oxLDL in DCs.

\section{Acknowledgements}

The present study was funded by the Natural Science Foundation of China (grant no. 81200214/H0215).

\section{References}

1. Hansson GK and Hermansson A: The immune system in atherosclerosis. Nat Immunol 12: 204-212, 2011.

2. Libby P, Ridker PM and Hansson GK: Progress and challenges in translating the biology of atherosclerosis. Nat Immunol 473: 317-325, 2011.

3. Niessner A and Weyand CM: Dendritic cells in atherosclerotic disease. Clin Immunol 134: 25-32, 2010.

4. Han J, Lee Y, Yeom KH, Nam JW, Heo I, Rhee JK, Sohn SY, Cho Y, Zhang BT and Kim VN: Molecular basis for the recognition of primary microRNAs by the Drosha-DGCR8 complex. Cell 125: 887-901, 2006.

5. Sayed D, Hong C, Chen IY, Lypowy J and Abdellatif M: MicroRNAs play an essential role in the development of cardiac hypertrophy. Circ Res 100: 416-424, 2007.

6. Tatsuguchi M, Seok HY, Callis TE, Thomson JM, Chen JF, Newman M, Rojas M, Hammond SM and Wang DZ: Expression of microRNAs is dynamically regulated during cardiomyocyte hypertrophy. J Mol Cell Cardiol 42: 1137-1141, 2007.

7. Thum T, Galuppo P, Wolf C, Fiedler J, Kneitz S, van Laake LW, Doevendans PA, Mummery CL, Borlak J, Haverich A, et al: MicroRNAs in the human heart: a clue to fetal gene reprogramming in heart failure. Circulation 116: 258-267, 2007.

8. van Rooij E and Olson EN: microRNAs put their signatures on the heart. Physiol Genomics 31: 365-366, 2007.

9. van Rooij E and Olson EN: MicroRNAs: powerful new regulators of heart disease and provocative therapeutic targets. J Clin Invest 117: 2369-2376, 2007.

10. van Rooij E, Sutherland LB, Liu N, Williams AH, McAnally J, Gerard RD, Richardson JA and Olson EN: A signature pattern of stress-responsive microRNAs that can evoke cardiac hypertrophy and heart failure. Proc Natl Acad Sci USA 103: 18255-18260, 2006. 
11. Busch $\mathrm{M}$ and Zernecke $\mathrm{A}$ : microRNAs in the regulation of dendritic cell functions in inflammation and atherosclerosis. J Mol Med (Berl) 90: 877-885, 2012.

12. Lagos-Quintana M, Rauhut R, Yalcin A, Meyer J, Lendeckel W and Tuschl T: Identification of tissue-specific microRNAs from mouse. Curr Biol 12: 735-739, 2002.

13. O'Connell RM, Rao DS, Chaudhuri AA, Boldin MP, Taganov KD, Nicoll J, Paquette RL and Baltimore D: Sustained expression of microRNA-155 in hematopoietic stem cells causes a myeloproliferative disorder. J Exp Med 205: 585-594, 2008.

14. Lu F, Weidmer A, Liu CG, Volinia S, Croce CM and Lieberman PM: Epstein-Barr virus-induced miR-155 attenuates NF-kappaB signaling and stabilizes latent virus persistence. J Virol 82: 10436-10443, 2008

15. Shinmei S, Sakamoto N, Goto K, Sentani K, Anami K, Hayashi T, Teishima J, Matsubara A, Oue N, Kitadai Y and Yasui W: MicroRNA-155 is a predictive marker for survival in patients with clear cell renal cell carcinoma. Int J Urol 20: 468-477, 2013.

16. Sun Y, Wang M, Lin G, Sun S, Li X, Qi J and Li J: Serum microRNA-155 as a potential biomarker to track disease in breast cancer. PLoS One 7: e47003, 2012.

17. Nazari-Jahantigh M, Wei Y, Noels H, Akhtar S, Zhou Z, Koenen RR, Heyll K, Gremse F, Kiessling F, Grommes J, et al: MicroRNA-155 promotes atherosclerosis by repressing Bcl6 in macrophages. J Clin Invest 122: 4190-4202, 2012.

18. Wei Y, Zhu M, Corbalán-Campos J, Heyll K, Weber C and Schober A: Regulation of Csf1r and Bcl6 in macrophages mediates the stage-specific effects of microRNA-155 on atherosclerosis. Arterioscler Thromb Vasc Biol 35: 796-803, 2015.

19. Zhu J, Chen T, Yang L, Li Z, Wong MM, Zheng X, Pan X, Zhang L and Yan H: Regulation of microRNA-155 in atherosclerotic inflammatory responses by targeting MAP3K10. PLoS One 7: e46551, 2012.

20. Donners MM, Wolfs IM, Stöger LJ, van der Vorst EP, Pöttgens CC, Heymans S, Schroen B, Gijbels MJ and de Winther MP: Hematopoietic miR155 deficiency enhances atherosclerosis and decreases plaque stability in hyperlipidemic mice. PLoS One 7: e35877, 2012

21. Tian FJ, An LN, Wang GK, Zhu JQ, Li Q, Zhang YY, Zeng A, Zou J, Zhu RF, Han XS, et al: Elevated microRNA-155 promotes foam cell formation by targeting HBP1 in atherogenesis. Cardiovasc Res 103: 100-110, 2014.

22. Zhu GF, Yang LX, Guo RW, Liu H, Shi YK, Wang H, Ye JS, Yang ZH and Liang X: miR-155 inhibits oxidized low-density lipoprotein-induced apoptosis of RAW264.7 cells. Mol Cell Biochem 382: 253-261, 2013.

23. Zhang YH, Xia LH, Jin JM, Zong M, Chen M and Zhang B: Expression level of miR-155 in peripheral blood. Asian Pac J Trop Med 8: 214-219, 2015.
24. Chen T, Yan H, Li Z, Jing T, Zhu W, Ge J, Zheng X, Pan X, Yan $\mathrm{H}$ and Zhu J: MicroRNA-155 regulates lipid uptake, adhesion/chemokine marker secretion and SCG2 expression in oxLDL-stimulated dendritic cells/macrophages. Int J Cardiol 147: 446-447, 2011

25. McCoy CE, Sheedy FJ, Qualls JE, Doyle SL, Quinn SR, Murray PJ and O'Neill LA: IL-10 inhibits miR-155 induction by toll-like receptors. J Biol Chem 285: 20492-20498, 2010.

26. Li P, Grgurevic S, Liu Z, Harris D, Rozovski U, Calin GA, Keating MJ and Estrov Z: Signal transducer and activator of transcription-3 induces microRNA-155 expression in chronic lymphocytic leukemia. PLoS One 8: e64678, 2013.

27. Rodriguez A, Vigorito E, Clare S, Warren MV, Couttet P, Soond DR, van Dongen S, Grocock RJ, Das PP, Miska EA, et al: Requirement of bic/microRNA-155 for normal immune function. Science 316: 608-611, 2007.

28. Volinia S, Calin GA, Liu CG, Ambs S, Cimmino A, Petrocca F, Visone R, Iorio M, Roldo C, Ferracin M, et al: A microRNA expression signature of human solid tumors defines cancer gene targets. Proc Natl Acad Sci USA 103: 2257-2261, 2006.

29. Faraoni I, Antonetti FR, Cardone J and Bonmassar E: miR-155 gene: a typical multifunctional microRNA. Biochim Biophys Acta 1792: 497-505, 2009.

30. van den Berg A, Kroesen BJ, Kooistra K, de Jong D, Briggs J, Blokzijl T, Jacobs S, Kluiver J, Diepstra A, Maggio E and Poppema S: High expression of B-cell receptor inducible gene BIC in all subtypes of Hodgkin lymphoma. Genes Chromosomes Cancer 37: 20-28, 2003.

31. Martin MM, Buckenberger JA, Jiang J, Malana GE, Nuovo GJ, Chotani M, Feldman DS, Schmittgen TD and Elton TS: The human angiotensin II type 1 receptor $+1166 \mathrm{~A} / \mathrm{C}$ polymorphism attenuates microRNA-155 binding. J Biol Chem 282: 24262-24269, 2007.

32. Yin Q, Wang X, McBride J, Fewell C and Flemington E: B-cell receptor activation induces $\mathrm{BIC} / \mathrm{miR}-155$ expression through a conserved AP-1 element. J Biol Chem 283: 2654-2662, 2008.

33. Lind EF, Millar DG, Dissanayake D, Savage JC, Grimshaw NK, Kerr WG and Ohashi PS: miR-155 upregulation in dendritic cells is sufficient to break tolerance in vivo by negatively regulating SHIP1. J Immunol 195: 4632-4640, 2015.

34. Santiago FS, Lowe HC, Bobryshev YV and Khachigian LM: Induction of the transcriptional repressor Yin Yang-1 by vascular cell injury. Autocrine/paracrine role of endogenous fibroblast growth factor-2. J Biol Chem 276: 41143-41149, 2001.

35. Martin-McNulty B, Tham DM, da Cunha V, Ho JJ, Wilson DW, Rutledge JC, Deng GG, Vergona R, Sullivan ME and Wang YX: 17 Beta-estradiol attenuates development of angiotensin II-induced aortic abdominal aneurysm in apolipoprotein E-deficient mice. Arterioscler Thromb Vasc Biol 23: 1627-1632, 2003. 